\section{Smooth Motion Planning for Car-Like Vehicles}

\author{
F. Lamiraux and J.-P. Laumond
}

\begin{abstract}
This paper presents a steering method for a car-like vehicle providing smooth paths subjected to curvature constraints. We show how to integrate this steering method in a global motion planning scheme taking obstacles into account. The main idea of the paper is to consider the car as a 4-D system from a kinematic point of view and as a 3-D system from a geometric point of view of collision checking. The resulting planned motions are guaranteed to be collision-free and $C^{2}$ between two cusp points.
\end{abstract}

Index Terms-Nonholonomic car-like robot, smooth motion planning.

\section{INTRODUCTION}

In the framework of motion planning for nonholonomic systems, the car-like vehicle has been the most investigated system. Numerous motion planners including obstacle avoidance capabilities are today available (e.g., [3], [4], [17], [15], [8], [21], [11], [24], [29]). All these approaches consider the car-like vehicle as a three-dimensional (3-D) system moving in the plane and subjected to constraints on the curvature (in addition to the nonholonomic constraint of rolling without slipping). The pioneering work by Dubins [7] and then by Reeds and Shepp [23], showed that the minimal length ${ }^{1}$ paths for a car-like vehicle consist of a finite sequence of two elementary components: arcs of circle (with minimal turning radius) and straight line segments. From then, almost all of the proposed motion planners compute collision-free paths constituted by such sequences. As a result, the paths are piecewise $C^{2}$, i.e., they are $C^{2}$ along elementary components, but the curvature is discontinuous between two elementary components. To follow such paths, a real system has to stop at these discontinuity points in order to ensure the continuity of the linear and angular velocities.

To overcome this inconvenience several authors have proposed to smooth the sequences straight line-arc of circle by clothoids (e.g., [12], [9]). The paths are then $C^{2}$ between two cusp points. However, this approach raises another problem: clothoids do not have a closed form making the control of their shapes difficult and dangerous in the presence of obstacles. This smoothing technique usually affects the completeness of the motion planner. The only exception is the work appearing in [24].

In this paper, we propose to revisit the problem by considering a car as a 4-D system from a control point of view: the steering angle is a configuration variable. Such a system has been investigated from a control point of view (e.g., [30]) but without considering the obstacle avoidance problem.

After introducing the model of the car (Section II), we present the steering method which is derived from a method previously developed by the authors for a mobile robot pulling trailers (Section III). This approach guarantees the curvature of the path to be $C^{2}$ between cusp points. Then we show how to plug the steering method within two different nonholonomic motion planning schemes (Section IV). In the first scheme, the algorithm computes a collision-free holonomic path

Manuscript received October 24, 2000. This paper was recommended for publication by Associate Editor M. Overmars and Editor A. De Luca upon evaluation of the reviewers' comments. A shorter version of this paper appears in the 6th International Conference on Intelligent Autonomous Systems.

The authors are with LAAS-CNRS, Toulouse, France (e-mail: florent@ laas.fr; jpl@laas.fr).

Publisher Item Identifier S 1042-296X(01)08451-8.

${ }^{1}$ More precisely, the length here is the length of the path followed by the middle of the rear wheel axis. in $\mathbf{R}^{2} \times S^{1}$ (the nonholonomic constraints are ignored, only the obstacles of the environment are taken into account); then the path is approximated by a sequence of admissible paths computed with the steering method applied to the 4-D control system. In the second case, the local method is plugged into PRM (probabilistic roadmap planner): A graph is constructed by picking random free configurations and by connecting them by collision-free paths returned by our local method. To ensure the completeness of these schemes, the steering method has to account for the small-time controlability of the system: to connect configurations close to each other, the steering method has to produce paths that remain close to these configurations [25].

The main contribution of this paper is not to provide a completely new method, but to combine existing techniques from an adequate model of the car and to propose a practical well-grounded algorithm for planning collision-free paths such that the curvature is continuous between cusp points.

\section{CONTROlability OF A CAR AND ADMISSIBLE PATHS}

Control Model: The modeling of vehicles according to their locomotion systems is well understood (see [6]). Let us consider the system represented in Fig. 1. The distance between the reference point $(x, y)$ and the middle point of the driving wheels is assumed to be 1 . The orientation of the car is denoted by $\theta$. The configuration space $\mathcal{C}=\mathbf{R}^{2} \times\left(S^{1}\right)^{2}$ of this system is 4 -D. The two controls of a car are the velocity $v$ of the driving wheels and the time derivative $\omega$ of the steering angle $\zeta$. The steering angle is constrained by mechanical bounds $|\zeta| \leq \zeta_{\max }$. A configuration $X=(x, y, \theta, \zeta)$ is said to be admissible if $|\zeta|<\zeta_{\max }$. A car corresponds to the following control system.

$$
\left(\begin{array}{c}
\dot{x} \\
\dot{y} \\
\dot{\theta} \\
\dot{\zeta}
\end{array}\right)=\left(\begin{array}{c}
\cos \zeta \cos \theta \\
\cos \zeta \sin \theta \\
\sin \zeta \\
0
\end{array}\right) v+\left(\begin{array}{l}
0 \\
0 \\
0 \\
1
\end{array}\right) \omega .
$$

Applying the technique of the Lie bracket rank condition, such a system is proved to be small-time controlable at any point (see for instance [20]). This means that, starting from any configuration, for any time $T$, the domain reachable with bounded velocity $(|v|<1,|\omega|<1)$ and in time less than $T$ always contains a neighborhood of the starting configuration.

In a lot of path planning work, the steering angle is not a configuration variable. In this case, the model can be simplified as follows. By setting $\tilde{v}=v \cos \zeta$ and $\tilde{\omega}=v \sin \zeta$ we get the following 3-D control system:

$$
\left(\begin{array}{c}
\dot{x} \\
\dot{y} \\
\dot{\theta}
\end{array}\right)=\left(\begin{array}{c}
\cos \theta \\
\sin \theta \\
0
\end{array}\right) \tilde{v}+\left(\begin{array}{l}
0 \\
0 \\
1
\end{array}\right) \tilde{\omega} .
$$

This system looks like the kinematic model of the so-called unicycle. The main difference lies in the admissible control domains. Here the constraints on $\tilde{v}$ and $\tilde{\omega}$ are no longer independent. By setting $v_{\max }=$ $\sqrt{2}$ and $\zeta_{\max }=\pi / 4$ we get $0 \leq|\tilde{\omega}| \leq|\tilde{v}| \leq 1$. The curvature of the path should be smaller than 1 , whenever it is defined. The various existing motion planners for cars usually consider this 3-D model.

In the next section, we build a steering method based on the combination of canonical paths. The idea is to combine two paths passing by two different configurations to get a feasible path that goes from the first to the second configuration. We define now what we call a canonical path associated to a configuration. 


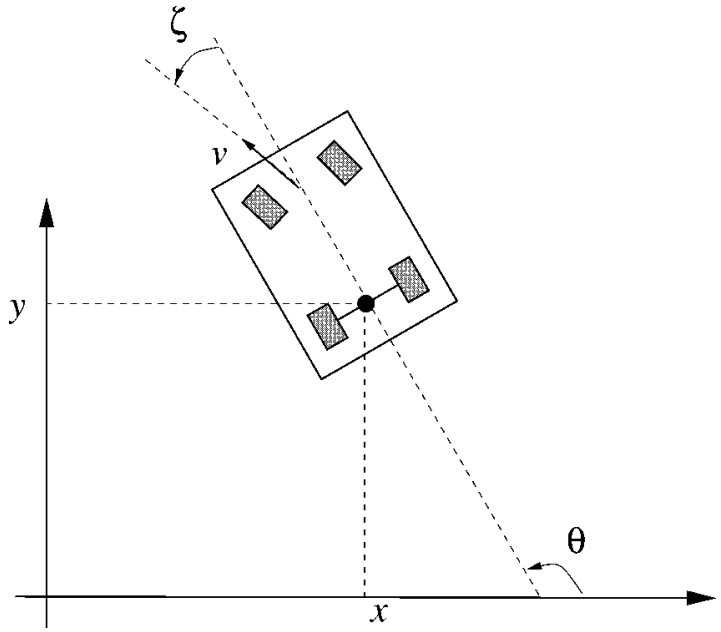

(a)

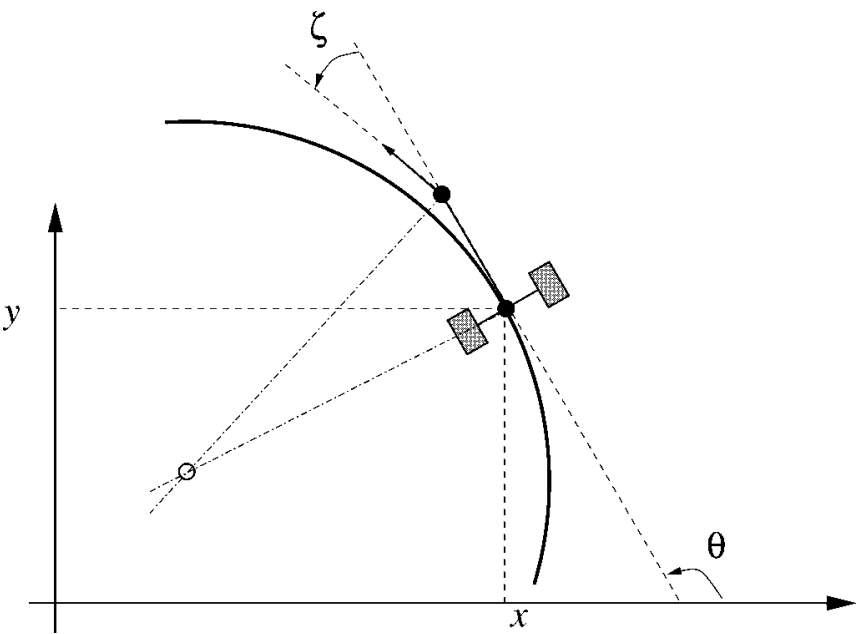

(b)

Fig. 1. Model of a car and a canonical curve.

Canonical Paths, Canonical Curves: Let us consider a sufficiently smooth path followed by the reference point. Simple computations show that the tangent to the path gives the orientation $\theta$ of the car, while the steering angle $\zeta$ is related to the curvature ${ }^{2} \kappa$ of the path by $\kappa=\tan \zeta$. These relations define a one-to-one mapping between the space of admissible configurations and $\mathbf{R}^{2} \times S^{1} \times[-1,1]$. In other word, any configuration can be parameterized by a vector $(x, y, \theta, \kappa)$ where $\kappa$ is the curvature defined above. Now, given a configuration $X=(x, y, \theta, \kappa)$, there exists a unique feasible path passing by $X$ and keeping $\kappa$ constant. This canonical path, denoted by $\Gamma(X, s)$, is obtained by integrating system (1) with $v=1$ and $\omega=0$ over the time interval $[0, s]$. The corresponding curve $\gamma(X, s)$ followed by the reference point $(x, y)$ is an arc of circle if $\kappa \neq 0$ and a straight line if $\kappa=0$. Let us notice that this curve is parameterized by arc-length $s . \gamma(X, s)$ is called the canonical curve associated to $X$. By construction we get the following property.

Property 1: The canonical path of an admissible configuration verifies the curvature constraint.

\section{A STEERING METHOD}

To any configuration $X$, the definition above associates a path passing by this configuration. We are now going to show how to

${ }^{2}$ This property derives from the notion of flatness recently introduced in control theory [10].

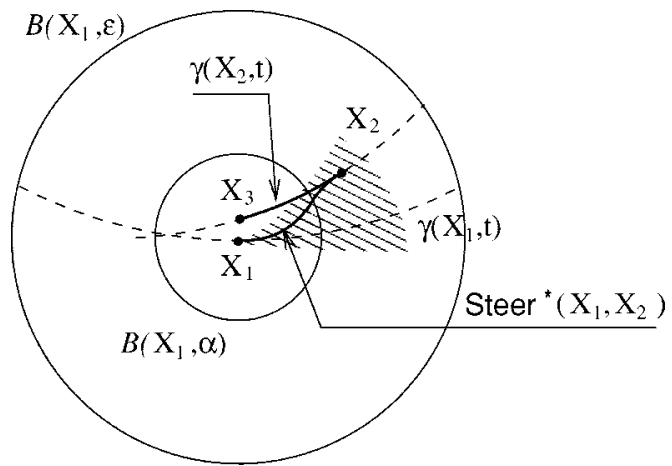

Fig. 2. Steer accounts for small-time controlability.

use these canonical curves to build a feasible path between two configurations.

We define a smooth increasing function $\alpha$ from $[0,1]$ into $[0,1]$ verifying: $\alpha(0)=0, \alpha(1)=1, \dot{\alpha}(0)=\ddot{\alpha}(0)=\dot{\alpha}(1)=\ddot{\alpha}(1)=0$. Let $X_{1}$ and $X_{2}$ be the initial and goal configurations, respectively.

It can be easily verified that the curve $P(t)=(1-\alpha(t)) \gamma\left(X_{1}, t\right)+$ $\alpha(t) \gamma\left(X_{2}, t-1\right)$ has the same position, tangent and curvature as $\gamma\left(X_{1}, t\right)$ for $t=0$ and as $\gamma\left(X_{2}, t-1\right)$ for $t=1$. Therefore, it corresponds to a feasible path $X(t)$ in $\mathcal{C}$ going from $X_{1}$ to $X_{2}$ when $t$ goes from 0 to 1 . The configurations along $X(t)$ are computed from the curve $P(t)$, the orientation of its tangent and its curvature using the relation $\zeta=\arctan \kappa$. The key point here is that $\kappa$ is continuous along $X(t)$.

This construction defines a steering method that allows the car to reach any configuration from any other one. However, this steering method is not suitable for integration in our collision-free scheme described below since it does not account for small-time controlability as defined now (see [25] for details).

Definition: A steering method Steer is said to account for small-time controlability if it satisfies the following property

$$
\begin{aligned}
& \forall \varepsilon>0, \exists \alpha>0, \forall\left(X_{1}, X_{2}\right) \in \mathcal{C}^{2} \\
& d\left(X_{1}, X_{2}\right)<\alpha \Rightarrow \operatorname{Steer}\left(X_{1}, X_{2}\right) \subset \mathcal{B}\left(X_{1}, \varepsilon\right)
\end{aligned}
$$

where

$d \quad$ distance in the configuration space $\mathcal{C}$ of the system; $\mathcal{B}\left(X_{1}, \alpha\right) \quad$ ball of radius $\alpha$ centered on $X_{1}$ (using distance $d$ );

$\operatorname{Steer}\left(X_{1}, X_{2}\right)$ path followed by the steering method between $X_{1}$ and $X_{2}$.

To account for small-time controlability, it can be easily stated that a steering method has to generate cusp points. In [14], we show how to build a steering method accounting for small-time controlability using the above convex combination of canonical curves. We briefly recall here the main ideas of this construction. Let $X_{1}=\left(x_{1}, y_{1}, \theta_{1}, \kappa_{1}\right)$ and $X_{2}=\left(x_{2}, y_{2}, \theta_{2}, \kappa_{2}\right)$ be initial and final configurations. We define $M_{2}$ as the orthogonal projection of $\left(x_{2}, y_{2}\right)$ on $\gamma\left(X_{1}, t\right)$. We define $v_{2}$ the parameter of this projection on $\gamma\left(X_{1}, t\right): M_{2}=\gamma\left(X_{1}, v_{2}\right)$. Then we slightly modify the above construction of $P(t)$ as follows:

$$
P(t)=(1-\alpha(t)) \gamma\left(X_{1}, v_{2} t\right)+\alpha(t) \gamma\left(X_{2}, v_{2}(t-1)\right) .
$$

The corresponding path in $\mathcal{C}$, that we denote by

Steer $^{*}\left(X_{1}, X_{2}\right)(t)$, still represents a feasible path going from $X_{1}$ to $X_{2}$. Importantly, if $X_{2}$ is on the canonical path associated to $X_{1}$, Steer $^{*}\left(X_{1}, X_{2}\right)(t)$ is exactly the canonical path $\Gamma\left(X_{1}, t\right)$. The continuity of Steer* ${ }^{*}$ w.r.t. $X_{1}$ and $X_{2}$ enables us to prove that an open set around the canonical path $\Gamma\left(X_{1}, t\right)$ (the shaded area in Fig. 2) is reachable by Steer* ${ }^{*}$ without escaping the ball of radius $\varepsilon$ centered on $X_{1}$. Using now the continuity of $\Gamma(X, t)$ w.r.t. $X$, we establish that if 

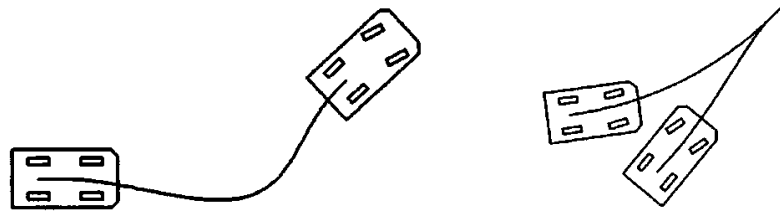

Fig. 3. Two paths generated by Steer. The first one (left) without cusp is a convex combination of the canonical curves associated to each configuration. The second one (right) is composed of a convex combination between the first configuration (bottom) and a cusp configuration and of the canonical path associated to the second configuration (top).

a configuration $X_{3}$ is close to $X_{1}, \Gamma\left(X_{3}, t\right)$ intersects the open set we have just defined close to $X_{1}$ and we can chose a configuration $X_{2}$ in this intersection. This process defines another steering method Steer as follows:

1) if $X_{3}$ is in the open set reachable by Steer*, then Steer $\left(X_{1}, X_{3}\right)=\operatorname{Steer}^{*}\left(X_{1}, X_{3}\right)$;

2) otherwise Steer $\left(X_{1}, X_{3}\right)$ is composed of two subpaths. The first one goes from $X_{1}$ to $X_{2}$ following Steer* $\left(X_{1}, X_{2}\right)$ between $X_{1}$ and $X_{2}$. The second one goes from $X_{2}$ to $X_{3}$ following $\Gamma\left(X_{3}, t\right)$.

With this construction Steer can access a neighborhood of a configuration $X_{1}$ without escaping any given ball centered on $X_{1}$ (Fig. 2). It accounts for small-time controlability. As a consequence, if $X_{1}$ and $X_{3}$ are admissible configurations close enough, then $\zeta_{1}$ and $\zeta_{3}$ are close enough in $]-\zeta_{\max }, \zeta_{\max }$ [ to guarantee that all the configurations $(x, y, \theta, \zeta)$ generated by $\operatorname{Steer}\left(X_{1}, X_{3}\right)$ are admissible, i.e., they verify $\zeta \in]-\zeta_{\max }, \zeta_{\max }[$. Gathering this result with the curvature continuity result we get the following property.

Property 2: For two sufficiently close admissible configurations $X_{1}$ and $X_{3}$, all the configurations of the path $\operatorname{Steer}\left(X_{1}, X_{3}\right)$ are admissible. The path followed by the reference point is $C^{2}$ between $X_{1}$ and $X_{2}$ and between $X_{2}$ and $X_{3}$.

Remark: The collision-free path planning scheme we define later builds paths composed of sequences of subpaths generated by Steer. The continuity of the curvature between two subpaths ensures us that a real system can follow these paths without stopping between each subpaths.

Fig. 3 shows two examples of paths generated by Steer.

\section{Plug-In Steer In Two Motion Planning SchemeS}

The first path planning scheme works for any small-time controlable system. Introduced in [17] it consists in approximating a collision-free (holonomic) path by a sequence of collision-free admissible ones. It only requires a steering method accounting for small-time controlability (Definition in Section III).

\section{A. Approximation of a Holonomic Path}

Geometric Planner: The first step is to find a geometric path, that is a collision-free path that does not take into account the nonholonomic constraints. The car is viewed as a polygon moving freely in translation and rotation in $\mathbf{R}^{2}$ among obstacles. The configuration space of this system is then $\mathbf{R}^{2} \times S^{1}$. Numerous techniques are available to address the motion planning problem in that case [16]. Among them we chose the "distributed representation approach" [2] that leads to resolution-complete algorithms (such algorithms are guaranteed to find a solution when a solution exists at a given resolution when modeling the search space by a grid). This algorithm is based on the construction of a potential field over the configuration space, the global minimum of which is the goal configuration. This potential field is built from

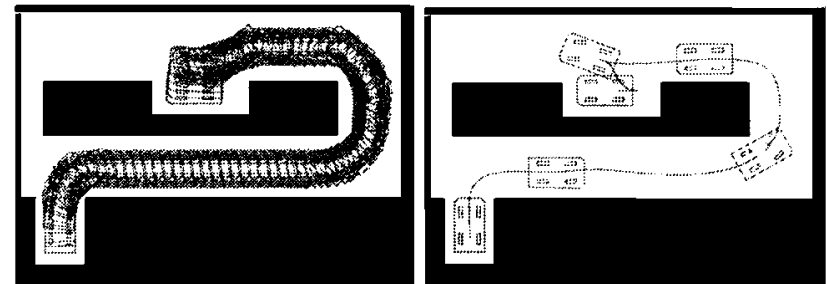

Fig. 4. A first geometric path and the feasible path generated by the approximation scheme with $\zeta_{\max }=60 \mathrm{deg}$.

two potential fields in the plane applied to two control points of the robot. Then the algorithm consists of an alternating sequence of gradient descent and a procedure filling the potential wells. Fig. 4 shows an example of a path computed using this method.

Approximation Step : Let us denote by $\Gamma_{\text {hol }}$ the geometric path computed in $\mathbf{R}^{2} \times S^{1}$ by the previous step. From now on, we consider $\Gamma_{\text {hol }}$ as a path in $\mathcal{C}$ by setting $\zeta=0$.

The approximation step recursively decomposes $\Gamma_{\text {hol }}$ as follows. A configuration $X$ is chosen in the middle of $\Gamma_{\text {hol }}$. This configuration is connected to $X^{\text {start }}$ and $X^{\text {goal }}$ using Steer, generating two feasible subpaths. Collision with obstacles and the curvature constraint $|\zeta|<$ $\zeta_{\max }$ are checked along these subpaths. If one of these constraints is violated, the corresponding subpath is discarded and a new subgoal is chosen on $\Gamma_{\text {hol }}$ between the beginning and the end of the discarded subpath.

Fig. 4 (right) shows the result of the approximation scheme performed on the holonomic path of Fig. 4 (left). All the computation are performed in a few seconds.

\section{B. Probabilistic Roadmap Approach}

We have plugged our local method in Move3D, a generic platform for path planning [27]. Move3D can solve path planning problems for any system as long as a geometric description of the system and a local steering method is provided. We have implemented the steering method defined in Section III within Move3D. Move3D plans path using the probabilistic roadmap approach [13]. Free configurations are randomly picked. A roadmap is built by connecting to each other configurations between which the steering method returns a local path without collision. A path planning problem is solved once the initial and goal configurations lie in the same connected component of the roadmap.

Figs. 5 and 6 show paths computed for a car by Move3D. The maximal steering angle $\zeta_{\max }$ is 30 degrees in both cases.

\section{Convergence and Completeness}

The convergence of the approximation step is guaranteed to finish in finite time as soon as the holonomic path belongs to an open domain of the collision-free configuration space and the steering method accounts for small-time controlability as defined above. The completeness of the algorithm thus inherits from the completeness of the geometric planner: it is resolution complete.

The probabilistic roadmap approach is probabilistically complete (i.e., the probability of finding a path if one exists tends toward 1 when the searching time increases) if the steering method accounts for small-time controlability.

Smoothing Step: Both planning algorithm provide a sequence of elementary admissible paths computed by Steer. This sequence usually include useless maneuvers and detours. A smoothing step tries to connect pairs of configurations randomly chosen on the path using the steering method to shorten the first solution path.

Remark on Optimal Paths: The minimal length paths for the system 2 have been characterized by Reeds and Shepp [23]. This result is 


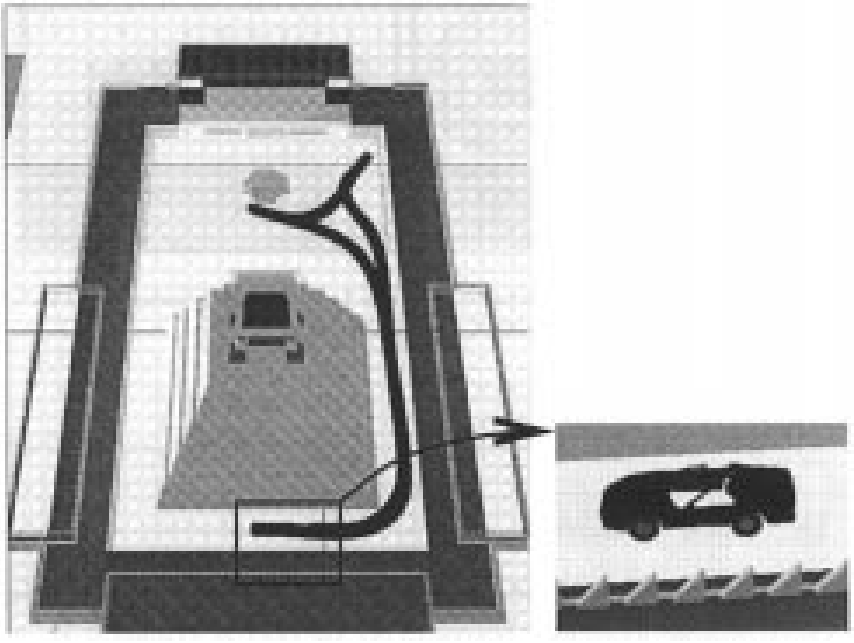

Fig. 5. A path computed by Move3D. The environment is a Mayan city with a pyramid. The car has to make a U-turn in a constrained corridor. Initial configuration is shown on the right. Final configuration is the same with opposite direction. This problem necessarily requires a long detour.

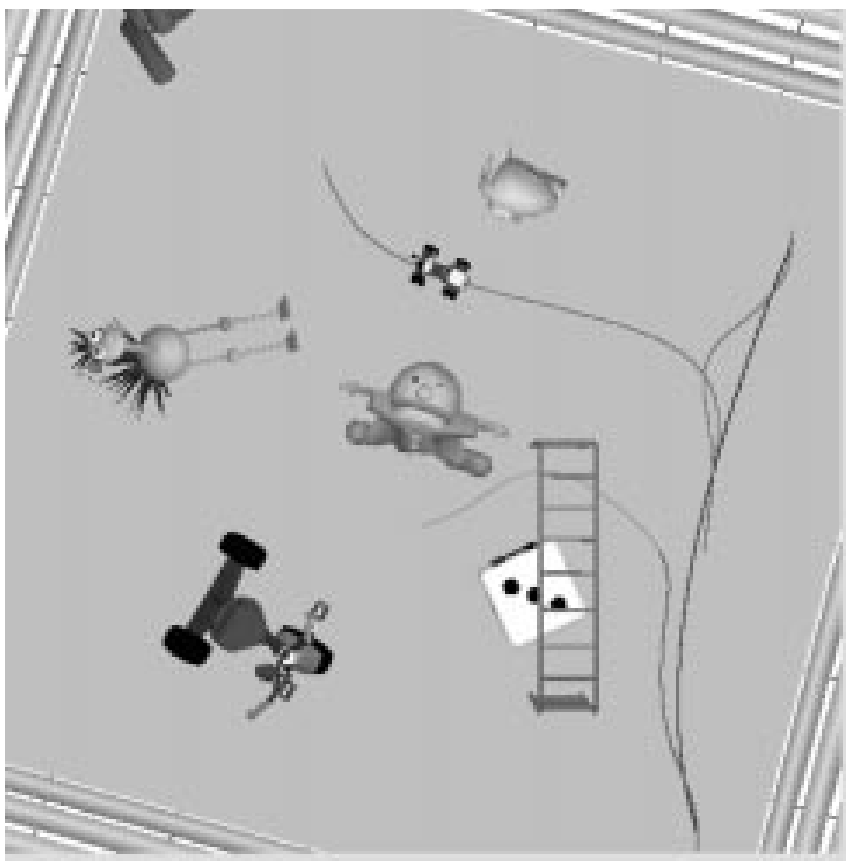

(a)

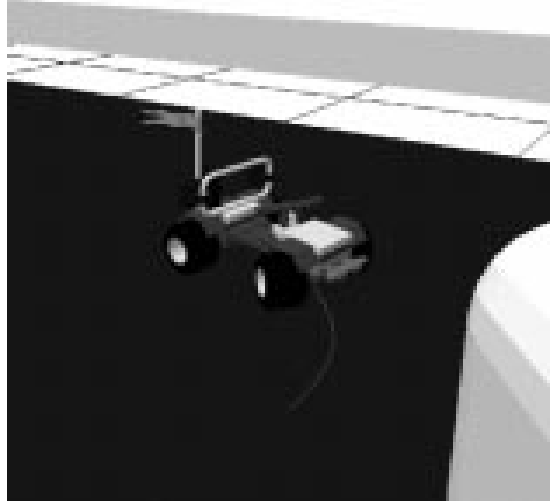

(b)

Fig. 6. A path computed for a car-like toy using Move3D. proven in the absence of obstacles. Adding obstacles give rise to a challenging problem: solutions exist via dynamic programming approaches [3], approximated approaches [26] or for special classes of obstacles [1], [5]. All these work do not consider any constraint on the continuity of the path curvature. Computing minimal length paths for the system 1 remains today an open problem even in the absence of obstacle [28]. Therefore the paths computed by the algorithm presented in this paper are not optimal. We just argue that they are satisfactory from a practical point of view.

\section{CONCLUSION}

A path for car-like robot is a finite sequence of curves linking cusp configurations. Between two cusps the curves should be sufficiently smooth to allow non zero velocity at any point. In other words, the curvature should be continuous on these curves. The main purpose of this paper is to propose an efficient steering method for a car-like vehicle that computes such piecewise smooth paths. Moreover, the proposed steering method has been integrated within two motion planning schemes. In the approximation scheme, the global solution path of Fig. 4 has been computed within a few seconds. In the probabilistic approaches (Figs. 5 and 6) the solution paths have been computed within a few seconds after a pre-processing time of a few minutes.

The main idea underlying the method proposed in this paper is to consider the car as a 4-D system. In such a way the constraint on the steering angle is treated as an obstacle. The approach avoids numerical issues such as the one arising in previous methods based on clothoids.

\section{ACKNOWLEDGMENT}

The authors are grateful to E. Sims for providing the picture of Fig. 6. Elements of environments of Fig. 5 (Mayan city) and Fig. 6 come from http://www.3dcafe.com.

\section{REFERENCES}

[1] P. K. Agarwal, P. Raghavan, and H. Tamaki, "Motion planning for a steering-constrained robot through moderate obstacles," in ACM Symp. Computat. Geom., 1995.

[2] J. Barraquand and J. C. Latombe, "Robot motion planning: A distributed representation approach," Int. J. Robot. Res., 1991.

[3] J. Barraquand and J.-C. Latombe, "On nonholonomic mobile robots and optimal maneuvering," Revue d'Intelligence Artificielle, vol. 3, no. 2, pp. 77-103, 1989.

[4] J. Barraquand and J. C. Latombe, "Nonholonomic multibody mobile robots: Controllability and motion planning in the presence of obstacles," in Algorithmica: Springer-Verlag, 1993, vol. 10, pp. 121-155.

[5] J. D. Boissonnat and S. Lazard, "A polynomial-time algorithm for computing a shortest path of bounded curvature amidst moderate obstacle," in ACM Symp. Computat. Geom., 1996.

[6] G. Campion, G. Bastin, and B. d'Andréa-Novel, "Structural properties and classification of kinematic and dynamic models of wheeled mobile robots," IEEE Trans. Robot. Automat., vol. 12, 1996.

[7] L. E. Dubins, "On curves of minimal length with a constraint on average curvature and with prescribed initial and terminal positions and tangents," Amer. J. Math., vol. 79, pp. 497-516, 1957.

[8] P. Ferbach, "A method of progressive constraints for nonholonomic motion planning," in IEEE Int. Conf. Robot. Automat., Minneapolis, MN, 1996, pp. 2929-2955.

[9] S. Fleury, P. Souères, J.-P. Laumond, and R. Chatila, "Primitives for smoothing mobile robot trajectories," IEEE Trans. Robot. Automat., vol. 11, pp. 441-448, 1995.

[10] M. Fliess, J. Lévine, P. Martin, and P. Rouchon, "Flatness and defect of nonlinear systems: Introductory theory and examples," Int. J. Contr., vol. 61, no. 6, pp. 1327-1361, 1995.

[11] T. Fraichard, "Dynamic trajectory planning with dynamic constraints: A 'state-time space' approach," in IEEE/RSJ Int. Conf. Intell. Robots Syst., Yokohama, 1993, pp. 1393-1400.

[12] Y. Kanayama and N. Miyake, "Trajectory generation for mobile robots," in Robotics Research. Cambridge, MA: MIT Press, 1986, vol. 3, pp. 333-340. 
[13] L. Kavraki, P.Švestka, J.-C. Latombe, and M. H. Overmars, "Probabilistic roadmaps for path planning in high-dimensional configuration spaces," IEEE Trans. Robot. Automat., vol. 12, 1996.

[14] F. Lamiraux, S. Sekhavat, and J.-P. Laumond, "Motion planning and control for Hilare pulling a trailer," IEEE Trans. Robot. Automat., vol. $15,1999$.

[15] J. C. Latombe, "A fast path planner for a car-like indoor mobile robot," in 9th Nat. Conf. Artif. Intell., AAAI, Anaheim, CA, July 1991, pp. 659-665.

[16] - Robot Motion Planning. Boston, MA: Kluwer Academic, 1991

[17] J.-P. Laumond, P. Jacobs, M. Taiïx, and R. Murray, "A motion planner for non holonomic mobile robots," IEEE Trans. Robot. Automat., vol. 10, pp. 577-593, 1994

[18] J.-P. Laumond, "Feasible trajectories for mobile robots with kinematic and environment constraints," in Int. Conf. Intell. Autonomous Syst., Amsterdam, The Netherlands, 1986.

[19] — , "Controllability of a multibody mobile robot," IEEE Trans. Robot. Automat., vol. 9, pp. 755-763, 1993.

[20] J.-P. Laumond, S. Sekhavat, and F. Lamiraux, "Guidelines in nonholonomic motion planning," in Robot Motion Planning and Control, J.-P. Laumond, Ed. New York: Springer-Verlag, 1998.

[21] B. Mirtich and J. Canny, "Using skeletons for nonholonomic motion planning among obstacles," in IEEE Int. Conf. Robot. Automat., Nice, France, 1992, pp. 2533-2540.

[22] R. M. Murray and S. Sastry, "Steering nonholonomic systems using sinusoids," in IEEE Int. Conf. Decision Contr., 1990, pp. 2097-2101.

[23] J. A. Reeds and R. A. Shepp, "Optimal paths for a car that goes both forward and backward," Pacific J. Math., vol. 145, no. 2, pp. 367-393, 1990.

[24] A. Scheuer and T. Fraichard, "Collision-free and continuous-curvature path planning for car-like robots," in IEEE Int. Conf. Robot. Automat., Albuquerque, NM, 1997.

[25] S. Sekhavat and J.-P. Laumond, "Topological property for collision-free nonholonomic motion planning: The case of sinusoidal inputs for chained form systems," IEEE Trans. Robot. Automat., vol. 14, 1998.

[26] J. Sellen et al., "Approximation and decision algorithms for curvatureconstrained path planning: A state-space approach," in Robotics: The Algorithmic Perspective, P. K. Agarwal et al., Eds: A.K. Peters, 1998.

[27] T. Siméon, J.-P. Laumond, and F. Lamiraux, "Move3D: A generic platform for path planning," in Int. Symp. Assembly and Task Planning, Fukuoka, Japan, May 2001.

[28] P. Souères and J.-D. Boissonnat, "Optimal trajectories for nonholonomic mobile robots," in Robot Motion Planning and Control, J.-P. Laumond, Ed. New York: Springer-Verlag, 1998.

[29] P. Svestka and M. Overmars, "Coordinated motion planning for multiple car-like robots using probabilistic roadmaps," in IEEE Int. Conf. Robot. Automat., Nagoya, Aichi, Japan, 1995.

[30] D. Tilbury, R. Murray, and S. Sastry, "Trajectory generation for the $n$-trailer problem using goursat normal form," IEEE Trans. Automat. Contr., vol. 40, 1995.

\section{Automatic Camera Calibration for a Multiple-Sensor Integrated Coordinate Measurement System}

\author{
Tzung-Sz Shen and Chia-Hsiang Menq
}

\begin{abstract}
An automatic camera calibration scheme that utilizes a coordinate measuring machine (CMM) and a novel camera calibration algorithm is presented in this paper for a multiple-sensor integrated coordinate measurement system. In the proposed calibration scheme, the touch probe tip carried by the CMM is employed to automatically generate high-precision calibration target points for camera calibration and sensor integration. A novel camera calibration algorithm with analytical formulations is developed to calibrate camera parameters in three stages without nonlinear minimization procedures. Simulations and experiments were performed to verify the proposed camera calibration algorithm. The precision of the automatic camera calibration scheme is also evaluated.
\end{abstract}

Index Terms-Camera calibration, coordinate measuring machine, multiple-sensor integration.

\section{INTRODUCTION}

Among three-dimensional (3-D) digitizing sensors, vision systems can simultaneously acquire thousands of data points over a large spatial range; therefore, capturing the global surface information of an object in real time is possible. In order to simultaneously achieve highspeed and high-precision coordinate acquisition, a multiple-sensor integrated coordinate measurement system can employ the vision sensor to rapidly acquire the initial global surface information [10]. The obtained information can be subsequently used to automatically guide the touch probe sensor or laser scanner for coordinate measurement with higher precision. For reliable and precise surface information acquisition, the accuracy of the vision system is essential.

When a vision system is used for 3-D coordinate measurement, camera calibration is usually required. The accuracy of the camera parameters, influenced by the calibration hardware as well as the calibration algorithms, has direct impact on the performance of the whole vision system. In this paper, an automatic camera calibration scheme that utilizes the coordinate measuring machine (CMM) and a novel calibration algorithm is developed for the multiple-sensor integrated coordinate measurement system. The automatic camera calibration scheme adopts the tip of the CMM probe to provide high-precision 3-D coordinates for camera calibration and to establish a common coordinate system for sensor integration. On the other hand, camera calibration techniques can be classified into: 1) linear methods (e.g., [1], [10]); 2) direct nonlinear minimization methods (e.g., [6]); and 3) multiple-stage methods (e.g., [2], [3], [11], [12]). Multiple-stage methods have become more popular because they are compromises between linear and direct nonlinear minimization methods. In the early stage, most camera parameters are obtained using closed-form solutions, and then iterative approaches are used to numerically solve remaining parameters and/or refine the parameters.

In multiple-stage methods, Tsai [11] first developed a two-stage technique under a radial alignment constraint (RAC). When the

Manuscript received April 7, 2000; revised November 27, 2000. This pape was recommended for publication by Associate Editor J. Ponce and Editor S. Hutchinson upon evaluation of the reviewers' comments. This work was supported by the National Science Foundation under Grant DMI-9713768.

The authors are with the Coordinate Metrology and Measurement Laboratory, Department of Mechanical Engineering, The Ohio State University, Columbus, OH 43210 USA (e-mail: shen.45@ osu.edu; menq.1@ osu.edu).

Publisher Item Identifier S 1042-296X(01)08621-9. 